\title{
Analyse of Measurement Based Admission Control Methods in IMS Networks and Simulation Principle
}

\author{
Erik Chromy*, Tomas Simoncik ${ }^{* *}$, Marek Holiencin ${ }^{* * *}$, and Ivan Baronak ${ }^{* * * *}$ \\ ${ }^{1}$ Slovak University of Technology in Bratislava, Faculty of Electrical Engineering and Information Technology, Bratislava, Slovakia
}

\begin{abstract}
This paper deals with the quality of service in IP Multimedia Subsystem networks. The evaluation of Quality o Service (QoS) is done through admission control methods. We analyse various methods, which are based on the traffic measurement. The approach that can be applied in finding the suitable admission control method while considering selected QoS parameters is described. Different important QoS parameters can be defined, however we decided to focus on the loss parameter. In our simulations we have used Simple Sum method. Based on the simulation results, we have used new algorithm. The main difference is that it uses the mechanism of time window for achieving more accurate traffic estimate. Algorithm Measured Sum with implementation of time window $T$ has better ability to predict the traffic fluctuation. Compared to the Simple Sum algorithm it achieves lower link utilisation, but also lower level of loss ratio.
\end{abstract}

\section{Introduction}

The IP Multimedia Subsystem (IMS) is an architectural framework for delivering Internet Protocol multimedia services [1]. IMS provides real-time multimedia sessions (VoIP, IPTV, conference session, etc.) [2]-[4] and non real-time multimedia sessions (Push to talk, Presence, Instant Messaging) over an IP network. Within the IMS framework the question of security, signalisation (H.248, MGCP, SIP and Diameter) and Quality of Service (QoS) [10], [11] are being discussed among the telecommunication companies, researchers and academics [12]-[15]. The goal of the IMS technology is to unify the mobile and fixed communication with IP technologies. This convergence could provide many options and services to users. IMS can provide interoperability between fixed and mobile networks and can also ensure the transfer of services.

The fact that IP belongs to the group of connectionless protocols causes multiple problems from the perspective of QoS. In this type of networks it is problematic to ensure acceptable levels of parameters like delay, jitter, or loss ratio. Solutions to this are therefore being investigated.

Admission control (AC) is an important tool for ensuring the QoS in IP networks. AC can be defined as a set of principles used to protect the network from overloading and it is also responsible for efficient utilisation of network resources [18]. This type of control mechanism should be implemented mostly in access networks in the nodes where the traffic is aggregated. The AC methods replaced its predecessor, Connection Admission Control

\footnotetext{
*e-mail: chromy@ut.fei.stuba.sk

**e-mail: tomassimoncik@gmail.com

***e-mail: holiencin.marek@gmail.com

****e-mail: baronak@ut.fei.stuba.sk
}

(CAC), known from the ATM technology. Many scientific papers presenting the research on CAC [5]-[9].

The goals of admission control methods are: to guarantee the quality of the service for the newly accepted connection without limitation of the other existing connections, to decide quickly with minimal delay and efficient use of the bandwidth capacity.

The admission control methods are divided into two groups: PBAC (Parameter Based Admission Control) and MBAC (Measurement Based Admission Control). PBAC methods are based on the technical parameters of the network in which they are used.

- PBAC methods: are based on the technical parameters of a network or data source (e.g. peak rate of data flow or efficient use of bandwidth),

- MBAC methods: uses real-time network traffic measurement. Based on this measurement and QoS requirements the MBAC method decides about new connection. For this measurement it is necessary to have information about the available bandwidth and some information about the data source that requests the connection. Compared to the PBAC methods, MBAC methods uses network resources more effectively

In IMS network the AC algorithms are implemented in the RACS (Resource and Admission Control Sub-system) block [16], [17]. RACS reserves appropriate resources and allow the requirement assuming policies, and required resources in the transport network are available. RACS provides the means for operators to promote admission control and option to set policies for service providers. In providing QoS influencing factor is admission control, providing to permit or to denial connection with allocating the required bandwidth. Element of RACS is responsible for admission control, while for defining its architecture 
is necessary to determine the possible QoS management functions in fixed networks.

The rest of this paper is organized as follows: Section 2 is devoted to the TISPAN RACS architecture. In Section 3 there are information about Measurement based admission control methods. The subsection 3.1 represents the most used MBAC algorithms. The section 4 brings information about simulation principle and results. In this section are information about Simple sum algorithm and Measured Sum algorithm with time window.

\section{RACS Architecture}

TISPAN (Telecommunications and Internet Converged Services and Protocols for Advanced Networks) architecture is based on the NGN (Next Generation Network) from ITU-T (International Telecommunication Union Telecommunication Standardization Sector). TISPAN architecture is modular and block based. This means that in the future it is possible to add additional subsystems to existing subsystems to, which would provide new types of services. The service layer consists of following parts:

- IMS core,

- PSTN/ISDN emulation subsystem,

- other multimedial subsystems and applications,

- subsystems that are required for application access, profile management, security management, routing databases,etc.

Figure 1 shows the functional architecture of TISPAN RACS [23]. This block is composed of two parts:

- Service-based Policy Decision Function (SPDF),

- Access-Resource and Admission Control Function (ARACF).

Transport layer consists of two interworking entities:

- Border Gateway Function (BGF),

- Resource Control and Enforcement Function (RCEF).

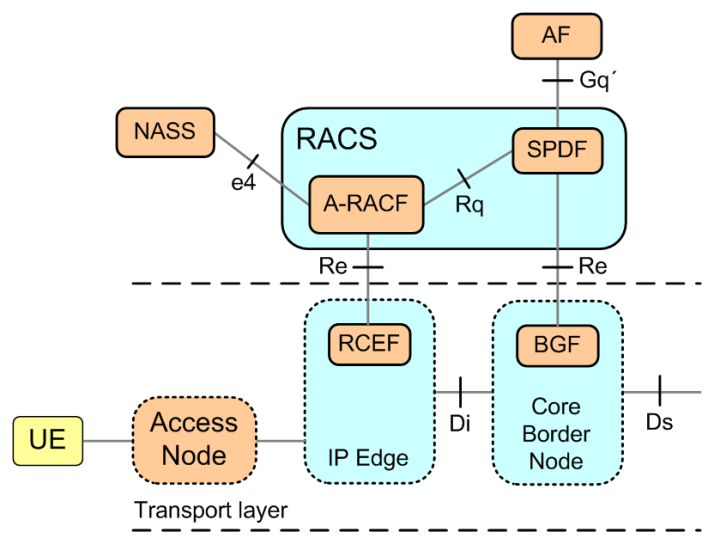

Figure 1. TISPAN RACS architecture

SPDF (Service Policy Decision Function) provides an interface for the service layer, while hiding the information about RACS and the network topology. The function of SPDF in the AC process is verifying, if the resource allocation request from AF complies with the defined rules. The local policy for is changed based on the incoming request. A change can be observed in the settings of QoS request for IP protocol. SPDF then locates the BGF and A-RACF, which will request the allocation of resources.

The A-RACF checks if the amount of requested bandwidth is compatible with the corresponding portion of the subscriber access profile, which may be received from the NASS over the $e 4$ reference point, and the amount of bandwidth remaining taking into account existing reservations. RACF can accept QoS requirements from SPDF through reference point $R q$, from RCEF (Resource Control and Enformcement Function) through reference point $R e$.

BGF (Border Gateway Function) provides interface between two IP transport domains (access and core network.)

\section{Measurement Based Admission Control}

The principle of Measurement Based Admission Control (MBAC) (Fig. 2) consist of three basic elements:

- admission decision algorithm,

- traffic estimator,

- resource estimator

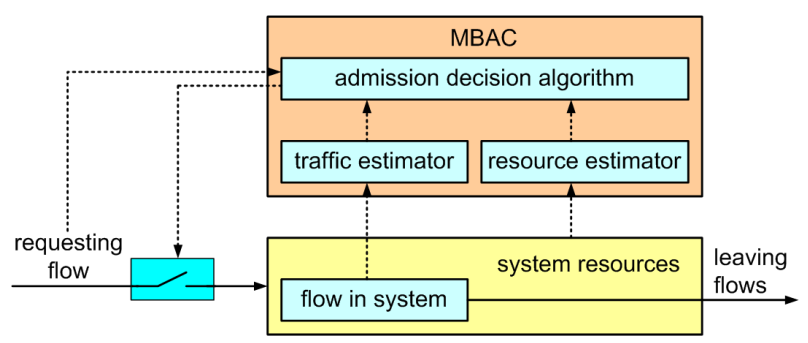

Figure 2. Measurement based admission control

MBAC algorithm measures the traffic and the amount of remaining system resources, e.g. available bandwidth, buffer size. Traffic estimator predicts the traffic in the system, its properties and the number of accepted data flows based on the conducted measurements. Resource estimator predicts the amount of spare resources in the system. MBAC algorithm decides if the requesting flow will be admitted, based on the input from the estimation elements [19]. From the basic definition of admission control it is clear, that the final decision is also based on the properties of the incoming flow, more specifically the requested QoS and its operational description.

\subsection{MBAC Algorithms}

\section{Simple Sum}

Simple Sum is the first of the admission control algorithms. It evaluates if the sum of admitted data flows is not exceeding the link capacity. New flow is admitted, if the following condition is met: 


$$
m_{i}+m<C
$$

$m$ is the measured load of existing traffic, $m_{i}$ is the peak rate of the new flow and $C$ is the bandwidth [20].

\section{Measured Sum}

Measured Sum is one of the most simple algorithms of MBAC methods. It measures the existing load in the system. The incoming data flow is accepted, if the following condition is satisfied:

$$
m_{i}+m<\alpha C
$$

$m$ is the measured load of existing traffic, $m_{i}$ is the peak rate of the new flow $i, \alpha$ is the required link utilisation from interval $(0,1)[21]$ and $\alpha . C$ is the overall bandwidth [bit/s]. MBAC methods are not suitable when the delay is too big. This occurs with a high link utilisation. Therefore the required link utilisation $\alpha$ is used in the decision making process.

\section{Hoeffding Bound}

The basic principle of the Hoeffding Bound algorithm is calculation of the equivalent bandwidth of actual traffic $C_{H}$. If the peak rate of the new flow $p$ and equivalent bandwidth of actual traffic summation is smaller than the link capacity, the incoming data flow will be accepted. Otherwise it will be rejected, according to the equation:

$$
C_{H}+p \leq C
$$

The equivalent bandwidth of actual traffic is calculated as follows:

$$
C_{H}=v+\sqrt{\frac{\ln \left(\frac{1}{\varepsilon}\right) \sum_{i=1}^{n}\left(p_{i}\right)^{2}}{2}}
$$

Where $n$ is the number of acceted flows, $v$ is the value of current traffic, estimated by the exponential averaging measurement mechanism, $p_{i}$ the peak rate of the admitted data flow $i, \epsilon$ is the required loss [22].

After accepting the new flow, the load estimation is increased by the value $v+p$.

\section{Tangent at Peak}

Algorithm Tangent at Peak uses a similar principle as the previous one. The following equation describes the new data flow acceptance criterion:

$$
n p\left(1-e^{-s p}\right)+e^{-s p} v \leq C
$$

where $n$ is the number of accepted flows.

\section{Tangent at Origin}

Algoritm Tangent at Origin is described with the following equation:

$$
e^{-s p} v \leq C
$$

\subsection{Traffic Estimation Mechanisms}

The measurement mechanisms used for estimating the traffic load are an important tool when dealing with MBAC methods. The obtained output contains information essential for making a decision about the new incoming data flow.

\section{Time Window}

Time window is a measurement mechanism used in the MS algorithm. It uses the time window for measuring the network traffic load. The principle of this mechanism is shown in Figure 3. In every sampling period $S$ the average load of the system is calculated. A new estimated load is set in the end of every time window $T$ as the highest calculated average of the current time window. In case of admitting a new flow to the system, the estimate increases by its value. If the new calculated average is higher than the estimated load, the estimated value is changed to match the value of the calculated average. In the end of every time window $T$ is the estimate set as the current load measured in the previous time window [15].

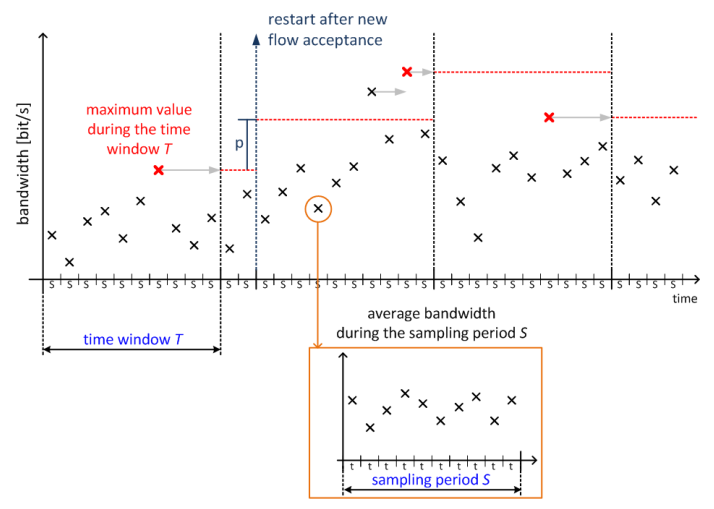

Figure 3. Measurement based admission control

\section{Simulation principle}

The aim of this part of the paper is describing a process of AC method simulation. This outline will present methods Simple Sum and Measured Sum.

The specification of RACS in TISPAN architecture does not define any specific AC method to be used. Choosing the most appropriate approach is left for the network administrator or system designer and is done based on the traffic characteristics and system type.

For simulating the admission control methods, program Matlab has been used. Matrix of dimensions $n x$ $n$ is generated to be used as a source of input data, representing the incoming traffic. Every line of the matrix corresponds to one data flow with a variable data rate, while the columns represent time $t$.

Each value stored in the matrix represents data rate $r_{p}$ in bits per second and is generated using a random number generator with uniform distribution. Peak rate of the data flows is set for $64 \mathrm{kbit} / \mathrm{s}$ (data flow range 0-64 kbit/s 


\begin{tabular}{c||ccccc|}
$n * n$ & $t_{1}$ & $t_{2}$ & $t_{3}$ & $\ldots$ & $t_{n}=T_{s}$ \\
\hline flow 1 & $r_{p 11}$ & $r_{p 12}$ & $r_{p 13}$ & $\ldots$ & $r_{p 1 n}$ \\
flow 2 & $r_{p 21}$ & $r_{p 22}$ & $r_{p 23}$ & $\ldots$ & $r_{p 2 n}$ \\
flow 3 & $r_{p 31}$ & $r_{p 32}$ & $r_{p 33}$ & $\ldots$ & $r_{p 3 n}$ \\
$\vdots$ & $\vdots$ & $\vdots$ & $\vdots$ & $\vdots$ & $\vdots$ \\
\hline flow j & $r_{p j 1}$ & $r_{p j 2}$ & $r_{p j 3}$ & $\ldots$ & $r_{p j n}$ \\
$\vdots$ & $\vdots$ & $\vdots$ & $\vdots$ & $\vdots$ & $\vdots$ \\
\hline flow n & $r_{p n 1}$ & $r_{p n 2}$ & $r_{p n 3}$ & $\ldots$ & $r_{p n n}$ \\
\hline & $t_{1}$ & $t_{2}$ & $t_{3}$ & $\ldots$ & $t_{n}=T_{s}$
\end{tabular}

Figure 4. Representation of data flows winth matrix $\mathrm{n} x \mathrm{n}$

has been chosen to avoid computational complexity and doesn't influence the results). The Figure 5 shows an example of data flow with a variable data rate.

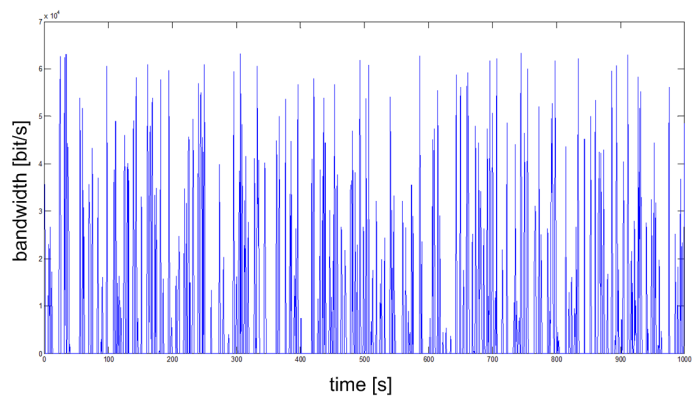

Figure 5. Example of single data flow

Individual data flows which form the entire traffic sequentially enter the network router, where MBAC algorithm is applied. The system bandwidth has been set as 10 Mbit/s. Network implementation is shown in the Figure 5.

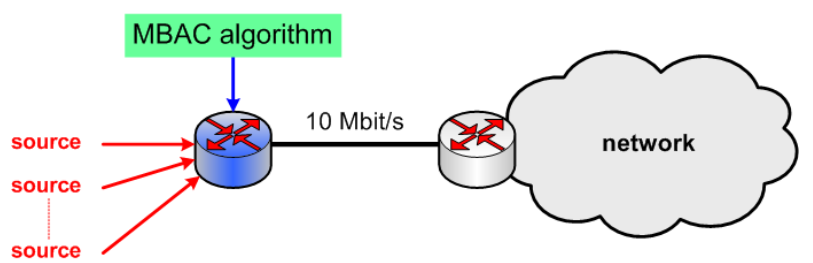

Figure 6. Example of single data flow

Parameters which are monitored during a chosen time interval are: link utilisation, number of transferred and lost bits, bit loss ratio and number of data flows admitted to the system. Every simulation has been repeated 200 times to ensure higher statistical relevance. Results presented in the paper are medians of the mentioned parameters.

\subsection{Simple Sum Algorithm}

Simple Sum is the basic and most simple MBAC algorithm. The Figure 6 shows the flowchart of this algorithm.

Matrix generated for the Simple Sum algorithm is presented in the Figure 7. Considering the simplicity of this

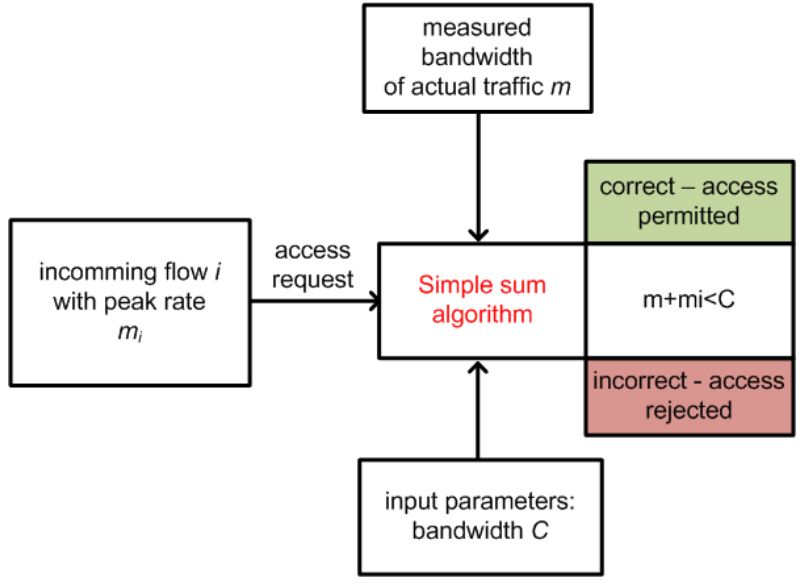

Figure 7. Flowchart of the Simple Sum algorithm

algorithm, it is possible to demonstrate the calculation directly on the input matrix. Accepting a new data flow is possible, if the sum of all flows already active in the network and the current measured value of the requesting flow is lower than the bandwidth (7). The result has to be only considered an estimate, as after admitting the new data flow into the system it is not guaranteed that the mentioned condition will still be met in $t+l$ (8). This situation can happen if data flow present in the network starts to transmit more data after a period of inactivity, or if the data rate of the newly admitted flow rises in time $t+1$. This scenario leads to exceeding the link capacity and increased loss ratio.

\begin{tabular}{c|cccccccc|} 
n*n & $t_{1}$ & $t_{2}$ & $t_{3}$ & $\ldots$ & $t$ & $t+1$ & $\ldots$ & $t_{n}=T_{s}$ \\
\hline flow 1 & $r_{p 11}$ & $r_{p 12}$ & $r_{p 13}$ & $\ldots$ & $r_{p 1 t}$ & $r_{p 1(t+1)}$ & $\ldots$ & $r_{p 1 n}$ \\
flow 2 & $r_{p 21}$ & $r_{p 22}$ & $r_{p 23}$ & $\ldots$ & $r_{p 2 t}$ & $r_{p 2(t+1)}$ & $\ldots$ & $r_{p 2 n}$ \\
\hline flow 3 & $r_{p 31}$ & $r_{p 32}$ & $r_{p 33}$ & $\ldots$ & $r_{p 3 t}$ & $r_{p 3(t+1)}$ & $\ldots$ & $r_{p 3 n}$ \\
\hline flow 4 & $r_{p 41}$ & $r_{p 42}$ & $r_{p 43}$ & $\ldots$ & $r_{p 4 t}$ & $r_{p 4(t+1)}$ & $\ldots$ & $r_{p 4 n}$ \\
\hline flow 5 & $r_{p 51}$ & $r_{p 52}$ & $r_{p 53}$ & $\ldots$ & $r_{p 5 t}$ & $r_{p 5(t+1)}$ & $\ldots$ & $r_{p 5 n}$ \\
\hline$\vdots$ & $\vdots$ & $\vdots$ & $\vdots$ & $\vdots$ & $\vdots$ & $\vdots$ & $\vdots$ & $\vdots$ \\
\hline flow j & $r_{p j 1}$ & $r_{p j 2}$ & $r_{p j 3}$ & $\ldots$ & $r_{p j t}$ & $r_{p j(t+1)}$ & $\ldots$ & $r_{p j n}$ \\
\hline$\vdots$ & $\vdots$ & $\vdots$ & $\vdots$ & $\vdots$ & $\vdots$ & $\vdots$ & $\vdots$ & $\vdots$ \\
\hline flow n & $r_{p n 1}$ & $r_{p n 2}$ & $r_{p n 3}$ & $\ldots$ & $r_{p n t}$ & $r_{p n(t+1)}$ & $\ldots$ & $r_{p n n}$ \\
\hline & $t_{1}$ & $t_{2}$ & $t_{3}$ & $\ldots$ & $t$ & $t+1$ & $\ldots$ & $t_{n}=T_{s}$
\end{tabular}

Figure 8. Input matrix for Simple Sum algorithm

$$
\begin{gathered}
\sum_{n=1}^{j-1} r_{p_{n t}}+r_{p_{j t}}<C \\
\sum_{n=1}^{j} r_{p_{n(t+1)}}<C
\end{gathered}
$$

\subsection{Measured Sum Algorithm with Time Window}

Measured sum is an algorithm which is based on previously mentioned Simple Sum. The main difference is that it uses the mechanism of time window. The goal of this algorithm is to achieve more accurate traffic estimation. 
Table 1. Input simulation parameters

\begin{tabular}{ll}
\hline parameters & values \\
\hline bandwidth & $10 \mathrm{Mbit} / \mathrm{s}$ \\
simulation duration & $7000 \mathrm{~s}$ \\
number of flows & 7000 \\
measured section & $5500-6500 \mathrm{~s}$ \\
number of repeated simulations & 200 \\
peak rate & $64 \mathrm{kbit} / \mathrm{s}$ \\
\hline
\end{tabular}

Table 2. Input simulation parameters of Measured Sum algorithm

\begin{tabular}{ll}
\hline parameters & values \\
\hline$T$ & 50 \\
$S$ & $10 \mathrm{~s}$ \\
peak rate & 0.95 \\
\hline
\end{tabular}

Flowchart of Measured Sum algorithm is shown in the Figure 8 .

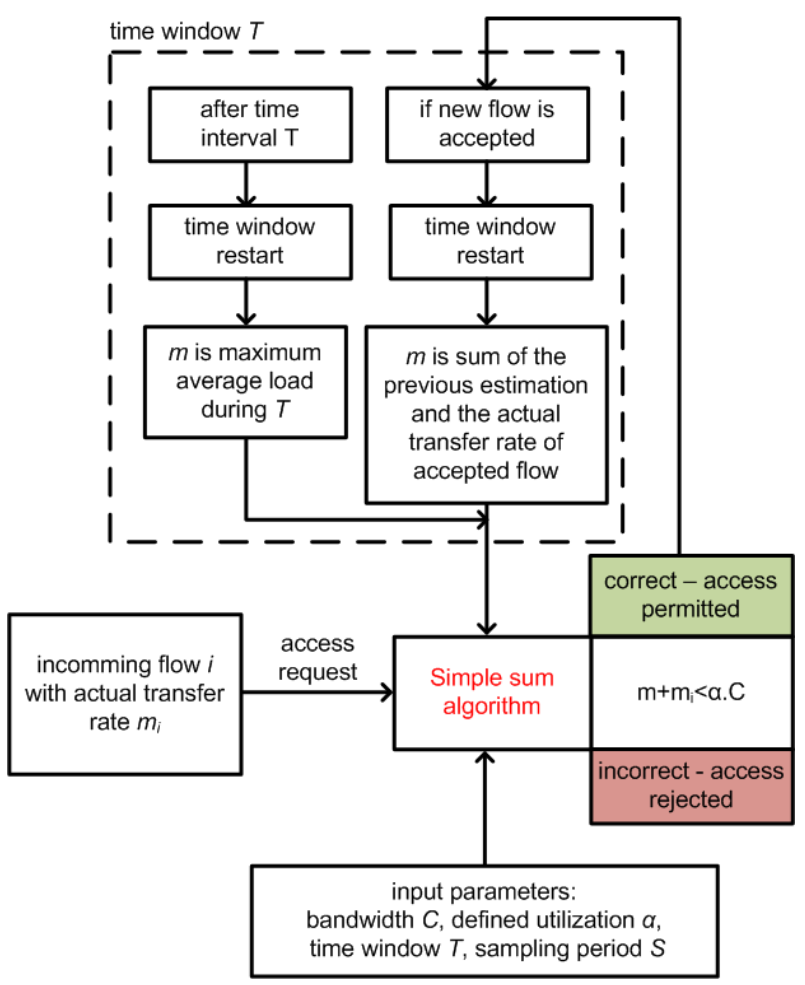

Figure 9. Flowchart of Measured Sum algorithm with time window $\mathrm{T}$

\subsection{Input Parameters of Simulated Algorithms and Results}

Parameters of the simulations and each MBAC algorithm are shown in Table 1 and 2 . In Table 3 the results are presented.

\subsection{Evaluation of Simulation Results}

In the simulations, the monitored parameters were: link utilisation, loss and number of flows admitted to the sys-
Table 3. Simulation results

\begin{tabular}{llll}
\hline algorithm & utilisation [\%] & loss [\%] & data flows \\
\hline Simple Sum & 100 & 5.30 & 1346 \\
Measured Sum & 95.72 & 0.80 & 1199 \\
\hline
\end{tabular}

tem. The key QoS parameter we focused on in evaluation was loss.

The Simple Sum algorithm is unable to predict traffic fluctuation, which is the reason for the loss ratio result of $5,3 \%$. It was, however, able to accept more data flows into the system than the second simulated method. The main advantage of this algorithm is its simplicity and low computational complexity, which may prove to be a key benefit in comparison with other methods.

Algorithm Measured Sum with implementation of time window $T$ has better ability to predict the traffic fluctuation. Compared to the Simple Sum algorithm it achieves lower link utilisation, but also lower level of loss ratio. Using this algorithm resulted in less smaller number of data flows admitted to the network. From the properties of Measured Sum, we can also say, that its computational complexity is higher than the one of Simple Sum algorithm. It is necessary for AC algorithms to be not only efficient, but also quick to make the necessary decisions. Otherwise it could lead to inefficient usage of network resources and throttled traffic.

\section{Conclusion}

In this paper, we focused on the admission control methods in IP Multimedia Subsystem. We analysed various methods that are based on the traffic measurements. Based on the Simple sum method, we have used admission control method that uses the mechanism of time window. This method has better ability to predict the traffic fluctuation. In our simulations we focused on the loss parameter. The goal is to achieve better traffic estimation. At the end of the paper we have compared the Simple Sum algorithm with the admission control method with the time window mechanism.

For future work, it is important to perform and test the simulations with different data sets (VoIP, IPTV and data) to compare the results of chosen QoS parameters and eventually choose the correct method to be used in RACS block. The right choice of AC method is a key factor in maintaining a balance between efficient link utilisation and providing the appropriate QoS parameter levels.

\section{Acknowledgement}

This article was created with the support of the Ministry of Education, Science, Research and Sport of the Slovak Republic within the KEGA agency project - 007STU4/2016 Progressive educational methods in the field of telecommunications multiservice networks and VEGA agency project - 1/0462/17 Modeling of qualitative parameters in IMS networks. 


\section{References}

[1] L. Lehman, IP Multimedia Subsystem: general aspects and migration perspectives (ITU Workshop on Developments regarding telecommunication network architectures and services, Uganda, 2012)

[2] P. Bazot, R. Huber, J. Kappel, B.S. Subramanian, E. Oguejiofor, B. Georges, C. Jackson, C. Martin and A.Sur, Developing SIP and IP Multimedia Subsystem (IMS) Applications. (IBM Corp., 2007) 684 pages

[3] M. Halas, and S. Klucik, Modelling the Probability Density Function of IPTV Traffic Packet Delay Variation. Advances in Electrical and Electronic Engineering 10 pp. 259-263 (2012)

[4] S. Klucik, and M. Lackovic, Modelling of H.264 MPEG2 TS Traffic Source. Advances in Electrical and Electronic Engineering 11 pp. 404-409 (2013)

[5] F. De Rango, M. Tropea, P. Fazio and S. Marano, Call admission control with statistical multiplexing for aggregate MPEG traffic in a DVBRCS satellite network. (GLOBECOM 05, IEEE Global Telecommunications Conference, St. Louis, USA, 2005)

[6] I. Baronak, and P. Kvackaj, A New CAC Method Using Queuing Theory. Radioengineering 17, pp. 62-74 (2008)

[7] R. Gibbens, F. Kelly, and P. Key, A Decision-Theoretic Approach to Call Admission Control in ATM Networks. IEEE Journal on Selected Areas in Communications 13 pp. 1101-1114 (1995)

[8] M. Kavacky, and I. Baronak, Evaluation of Two Statistical CAC Methods for Variable Bit Rate Traffic Sources. Journal of Electrical engineering 59, pp. 178186 (2008)

[9] P. Kvackaj, and I. Baronak, Evaluation of Three CAC Methods: Gaussian Approximation Method, Method of Effective Bandwidth and Diffusion Approximation Method. Journal of Electrical Engineering 57, pp. 360364 (2006)

[10] J. Frnda, M. Voznak, and L. Sevcik, Impact of packet loss and delay variation on the quality of real-time video streaming. Telecommunication Systems 62, pp. 265-275 (2016)

[11] L. Orcik, M. Voznak, J. Rozhon, F. Rezac, J. Slachta, H. Toral-Cruz, and J.C.-W. Lin, Prediction of Speech Quality Based on Resilient Backpropagation Artificial Neural Network. Wireless Personal Communications 96 pp. 5375-5389 (2017)

[12] Z. Bosternak, and R. Roka, Approach of the T-CONT allocation to increase the bandwidth in passive optical networks Radioengineering 26 pp. 954-960 (2017)

[13] F. Certik, and R. Roka, Possibilities for Advanced Encoding Techniques at Signal Transmission in the Optical Transmission Medium. Journal of Engineering 2016 Article number 2385372 (2016)

[14] I. Bestak, and M. Orgon, Performance measurement of encryption algorithms used in PLC devices. International Journal of Research and Reviews in Computer Science 2 (2011)

[15] J. Misurec, and M. Orgon, Modeling of power line transfer of data for computer simulation. International Journal of Communication Networks and Security 3 pp. 104-111 (2011)

[16] G. Horak, and C. Herhoffer, IP interconnection testing approach in a TISPAN IMS environment. (Proceedings of 2012 15th International Telecommunications Network Strategy and Planning Symposium, NETWORKS 2012, Rome, Italy 2012)

[17] A. Darvishan, K. Bamasian, H. Yeganeh, and P. Eghtedari, A practical NGN model by evaluation of various NGN solutions and its conformance with IMSTISPAN. (ICIME 2010 - 2010 2nd IEEE International Conference on Information Management and Engineering, Volume 1, Article number 5477462, 2010)

[18] E. Alipour, and K. Mohammadi, Adaptive Admission Control for Quality of Service Guarantee in Differentiated Services Networks. International Journal of Computer Science and Network Security 8 pp. 93-98 (2008)

[19] Y. Jiang, P. Emstad, A. Nevin, V. Nicola, and M. Fidler, Measurement-Based Admission Control for a Flow-Aware Network. ( IEEE, Italy, 2005) 8 pages

[20] S. Jamin, S. Shenker, and P. Danzig, Comparison of Measurement-based Admission Control Algorithms for Controlled-Load Service. (IEEE, Kobe, 1997)

[21] S. Jamin,P. Danzig, S. Shenker, and L. Zhang, A Measurement-based Admission Control Algorithm for Integrated Services Packet Networks (Extended Version). IEEE/ACM Transactions on Networking, 20 pages (1997)

[22] G. Yi-ran, W. Suo-ping, and W. Hai-ya, A structural comparison of measurement-based admission control algorithms. The Journal of China Universities of Post and Telecommunications 13 pp. 81 - 86 (2006).

[23] ETSI ES 282003 v3.5.1: Telecommunications and Internet converged Services and Protocols for Advanced Networking (TISPAN); Resource and Admission Control Sub-System (RACS): Functional Architecture (2011) 